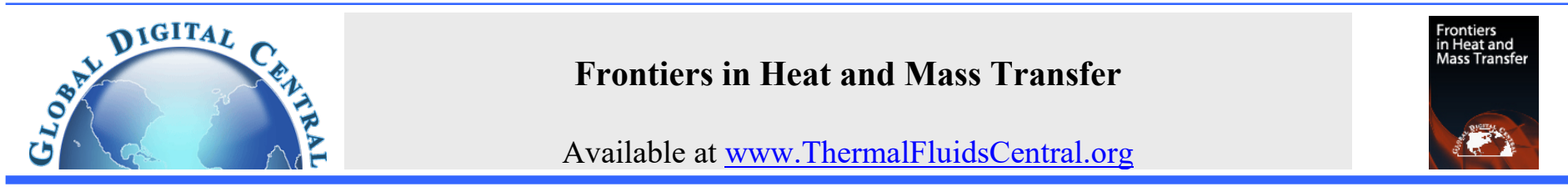

\title{
EFFECTS OF VARIABLE VISCOSITY ON HEAT AND MASS TRANSFER BY MHD MIXED CONVECTION FLOW ALONG A VERTICAL CYLINDER EMBEDDED IN A NON-DARCY POROUS MEDIUM
}

\author{
Saddam Atteyia Mohammad* \\ Mechanical Engineering Department, College of Engineering, University of Mosul, Mosul 41002, Iraq
}

\begin{abstract}
An analysis was performed to study the effects of variable viscosity on steady, laminar, hydromagnetic simultaneous heat and mass transfer by mixed convection flow along a vertical cylinder embedded in a non-Darcy porous medium. The analysis was performed for the case of power-law variations of both the surface temperature and concentration. The viscosity of the fluid is assumed to be an inverse linear function of temperature. Certain transformations were employed to transform the governing differential equations to non-similar form. The transformed equations were solved numerically by finite difference method. The entire regime of mixed convection was studied. From this study it can be concluded that increasing the values of power law index, curvature parameter and buoyancy ratio leads to enhance the local Nusselt and Sherwood numbers. The local Nusselt and Sherwood numbers weaken as the inertia effect parameter and the square of the Hartmann number increases. The raise in the value of the Lewis number decreases the rate of heat transfer while increases the rate of mass transfer. For lower values of viscosity, the heat transfer increased for both gases and liquids, while the mass transfer decreased for gases and increased for liquids.
\end{abstract}

Keywords: Heat and mass transfer, Mixed convection, Non-similarity solution, Porous medium, variable viscosity, vertical cylinder.

\section{INTRODUCTION}

During the past few decades the study of convective heat and mass transfer from different surfaces embedded in a saturated porous medium has got great attention. The motivation of this interest is the applications in thermal engineering systems (Flilihi et al., 2019). For example, thermal insulation, petroleum industries, nuclear engineering, geothermal systems, drying of porous solids and many other applications. In this field of studies, it's well known that, Darcy law is restricted by slow fluid velocity. Therefore, non-Darcian effects should be included in the mathematical formulation of the problem to get results more close to the real situations. These non-Darcian effects included inertia effects, thermal dispersion, boundary viscous resistance and porosity variation near the wall. Non-Darcy effects on flow and heat transfer characteristics in porous medium for different convection types and for different geometries can be found in earlier reports or studies. Generally, it can be infer from these studies that both thermal dispersion and variable porosity effects tend to increase the heat transfer rate, while boundary effects and flow inertia tend to decrease the rate of heat transfer. Due to these non-Darcian phenomenas it was found that the velocity and temperature profiles are altered considerably as compared to those estimated by Darcy law (Chen, 1998). In many situations it is adequate to regard the viscosity as constant. However, when a fluid layer is undergo to thermal gradients it may be rightly to take into account the variation of viscosity with temperature (Richardson and Straughan, 1993).

\footnotetext{
*Corresponding author.Email: saddamatteyia@uomosul.edu.iq
}

Studying of magneto hydrodynamic MHD flow and heat and mass transfer in different geometries has received good attention from researchers. This is because the effect of magnetic field on the flow control and performance of many systems using electrically conducting fluids like water mixed with little acid, liquid metals and others. Magneto hydrodynamic flow problems have become more important in industry. For instance, many metallurgical processes such as drawing, annealing and tinning of copper wires involve cooling of continuous strips or filaments by drawing them in an electrically conducting fluid in the presence of a magnetic field. The rate of cooling can be controlled in these processes which can be affect the properties of the final product (Amanulla et al., 2018; Ganapathirao et al., 2019). Study the effect of the magnetic field on the flow through porous medium is motivated from the fact that the fluids are electrically conducting in geothermal regions and can be significantly influenced by the magnetic field. Furthermore, magnetic field effects are encountered in various technological applications like metal casting, nuclear reactor coolers, geothermal energy extraction, purification of molten metals and many others (Ganapathirao et al., 2019). Below there are some of the studies about convection heat transfer over vertical cylinder.

First let us started with natural convection. Chen and Horng, (1999) studied the free convection along a vertical cylinder embedded in a thermally stratified non-Darcy porous medium. Hossain et al., (1999) study the effect of heat and mass transfer on the non-Darcy free convection flow along a vertical permeable cylinder. Takhar et al., (2002) analyzed the problem of free convection flow over a vertical 
cylinder embedded in non-Darcy thermally stratified high porosity medium. Chamkha et al., (2004) considered steady natural convection adjacent to an isothermal vertical circular cylinder in the presence of the thermophoresis particle deposition effect. Rani and Kim, (2008) study the influence of temperature-dependent viscosity and prandtl number on unsteady laminar natural convection flow along a vertical cylinder. Chamkha et al., (2011) investigate the heat and mass transfer by free convection over a vertical cylinder for a temperature-dependent fluid viscosity in the presence of radiation and chemical reaction effects. Shakeri et al., (2012) presented an analysis for natural convection flow adjacent to a vertical cylinder. Variable surface temperature condition was considered in this study. Furthermore, the fluid solid matrix are assumed to be in local thermal non-equilibrium, thus, two models of temperature for heat transfer is applied. Rashad et al., (2014) presented a study to investigate the free convection flow along a vertical cylinder embedded in a thermally stratified nanofluid-saturated non-Darcy porous medium. In the presence of thermal radiation a numerical solution of transient free convection MHD flow over a vertical cylinder of thermal and mass diffusion was presented by Reddy (2014). El-Kabeir et al., (2014) study non-Darcy free convection flow adjacent to a vertical cylinder embedded in a porous medium saturated with nanofluids with thermal radiation. Ferdows et al., (2015) studied the similarity solution of natural convection flow of a nanofluid on a heated vertical cylinder embedded in a nanofluid-saturated porous medium. The problem of unsteady non-Darcy natural convection flow of a viscous incompressible fluid past a vertical cylinder with the effect of chemical reaction was investigated by Vasu et al., (2017).

Now let us review some of the papers on mixed convection over vertical cylinder. Merkin and Pop, (1987) study the problem of mixed convection boundary layer flow over a vertical circular cylinder. The effect of the thermal dispersion on the non-Darcy mixed convection flow on a vertical cylinder was studied by Kumari et al., (1993). Hooper et al., (1994) analyze the problem of mixed convection from an isothermal vertical cylinder using Darcy model. Aldoss et al., (1996) investigated non-Darcy mixed convection flow from a vertical cylinder. Another study for Aldoss (1996) considered MHD mixed convection flow along with non-Darcy model. The effect of steady non-uniform suction or injection on mixed convection boundary layer flow over a vertical heated or cooled permeable slender cylinder was studied numerically using the Darcy law by Kumari et al., (2007). Gorla and Hossain, (2013) study the mixed convection boundary layer flow past a vertical cylinder in a porous medium saturated with a nanofluid. For both cases of a heated and a cooled cylinder, the steady mixed convection flow over a vertical cylinder embedded in a nanofluid-saturated porous medium is studied by Rohni et al., (2013). Jafarian et al., (2016) studied numerically the problem of conjugate heat transfer of magneto hydrodynamic MHD mixed convection of nanofluid over a vertical slender hollow cylinder embedded in a high porosity porous medium. The problem of steady mixed convection flow on a cooled vertical permeable circular cylinder was studied by Shu et al., (2017). Mohammad (2019) analyze the problem of simultaneous heat and mass transfer by steady mixed convection flow of an incompressible, viscous and electrically conducting fluid adjacent to a vertical cylinder embedded in a non-Darcy porous medium with heat source.

In this Work, It will be study the effects of variable viscosity on hydromagnetic coupled heat and mass transfer by combined convection flow over a vertical cylinder embedded in a non-Darcy porous medium. The entire regime of mixed convection will be studied (i.e. from pure free convection limit to pure forced convection limit). The high cost and the time required in experimental work has made the numerical modeling widely used in analyzing such problems. This type of studies or solutions are acceptable because of its capability of providing qualitative understanding of the physics of such problems. This study finds applications in the fields of geothermal and geophysical engineering such as extraction of geothermal energy, underground disposal of nuclear waste, the migration of moisture in fibrous insulation, and the spreading of chemical pollutant in saturated soil. To the best of my knowledge, such study has been overlooked in all of previous publications so that the results are novel and original.

\section{MATHEMATICAL ANALYSIS}

Consider the problem of two dimensional, steady, incompressible, laminar, non-Darcy, hydromagnetic coupled heat and mass transfer by mixed convection flow adjacent to a vertical circular cylinder embedded in a saturated porous medium as shown in Figure 1. It will be assumed that the fluid and solid matrix are in local thermal equilibrium. The fluid is Newtonian, electrically conducting and has constant properties except the density in the buoyancy terms of the balance of momentum equation that is approximated according to the Boussinesq approximation.

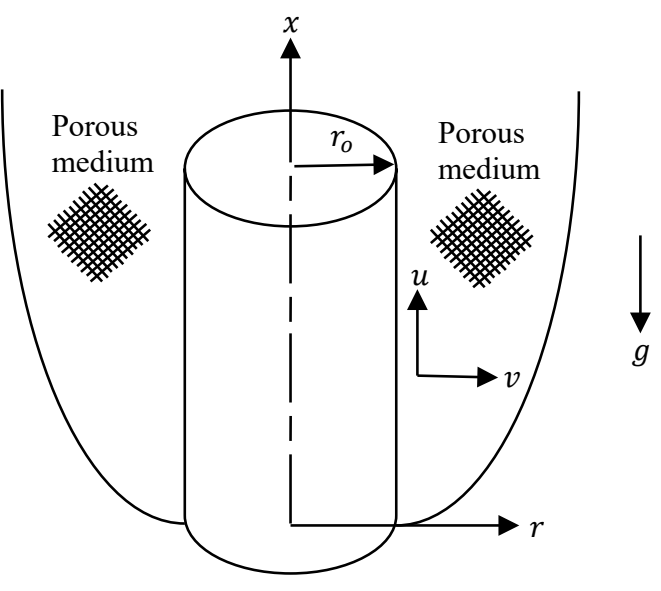

Fig. 1 Physical flow model and coordinate system.

The permeability and porosity of the porous medium are constant. The porous medium is non-deformable. Wall temperature and concentration vary according to power-law with the vertical distance. It will be assumed that the viscosity of the fluid is an inverse linear function of temperature. Under the above assumptions and using the Boussinesq and boundary layer approximations, the governing equations are given by

\subsection{Continuity Equation}

$\frac{\partial u}{\partial x}+\frac{\partial(r v)}{\partial r}=0$

Where $u$ is the velocity component in the $x$-direction and $v$ is the velocity component in the $r$-direction.

\subsection{Momentum Equation (Bejan, 1995)}

$\mu u=-K\left(\frac{\partial P}{\partial x}+\rho g+\frac{\sigma \beta_{o}^{2} u}{\emptyset}\right)-c \sqrt{K} \rho_{\infty} u^{2}$

$\mu v=-K\left(\frac{\partial P}{\partial r}\right)-c \sqrt{K} \rho_{\infty} v^{2}$

$c=1.75 /\left(\sqrt{150} \emptyset^{1.5}\right) \quad K=d^{2} \emptyset^{3} /\left[150(1-\emptyset)^{2}\right]$

Where $g$ is the gravitational acceleration; $P, \rho$ and $\mu$ are pressure, density and dynamic viscosity of the fluid respectively; $K$ and $\varnothing$ are the permeability and porosity of the porous medium respectively; $d$ is particle diameter; $c$ is constant; $\sigma$ and $\beta_{o}$ are the electrical conductivity of the fluid and the magnetic induction respectively. As stated above, 
fluid viscosity is assumed to be an inverse linear function of temperature (Rani and Kim, 2008; Chamkha et al., 2011; Jayanthi and Kumari, 2006)

$\frac{1}{\mu}=\frac{1}{\mu_{\infty}}\left[1+R\left(T-T_{\infty}\right)\right]=a\left(T-T_{e}\right)$

where $a=\frac{R}{\mu_{\infty}} \quad$ and $\quad T_{e}-T_{\infty}=-\frac{1}{R} \quad, a \neq 0, R \neq 0$

In Eq. (5) $T$ and $T_{\infty}$ are temperature and free stream temperature respectively. $a$ and $T_{e}$ are constants and their values depend on the thermal property of the fluid $R$ and the reference state. For liquids $a>0$ and for gases $a<0$. The viscosity of a gas usually increases with increasing temperature and it decreases for liquids. By differentiating Eqs. (2) and (3) with respect to $r$ and $x$ respectively, it can be eliminate pressure term from the resulting equations. Invoking the Boussinesq approximation

$\rho=\rho_{\infty}\left[1-\beta_{T}\left(T-T_{\infty}\right)-\beta_{C}\left(C-C_{\infty}\right)\right]$

where $C$ and $C_{\infty}$ are concentration and free stream concentration respectively. $\rho_{\infty}, \beta_{T}$ and $\beta_{C}$ are free stream density, thermal expansion coefficient and concentration expansion coefficient respectively. It will be assumed that within the boundary layer $(v \ll u, \partial v / \partial x \ll \partial u / \partial r)$, the final form of the momentum equation can be written as

$-\frac{1}{K}\left(\mu \frac{\partial u}{\partial r}+u \frac{\partial \mu}{\partial r}\right)-\frac{c \rho_{\infty}}{\sqrt{K}}\left(2 u \frac{\partial u}{\partial r}\right)-g \frac{\partial \rho}{\partial r}-\frac{\sigma \beta_{o}^{2}}{\emptyset} \frac{\partial u}{\partial r}=0$

\subsection{Energy Equation}

$u \frac{\partial T}{\partial x}+v \frac{\partial T}{\partial r}=\alpha\left[\frac{\partial 2 T}{\partial x^{2}}+\frac{1}{r} \frac{\partial}{\partial r}\left(r \frac{\partial T}{\partial r}\right)\right]$

where $\alpha$ is the thermal diffusivity.

\subsection{Concentration Equation}

$u \frac{\partial C}{\partial x}+v \frac{\partial C}{\partial r}=\frac{D}{r} \frac{\partial}{\partial r}\left(r \frac{\partial C}{\partial r}\right)$

where $D$ is the mass diffusivity.

\subsection{Boundary Conditions}

$r=r_{o} \quad v=0 \quad T=T_{w}(x)=T_{\infty}+A x^{n}$

$C=C_{w}(x)=C_{\infty}+B x^{n}$

$r \rightarrow \infty \quad u=U_{\infty} \quad T=T_{\infty} \quad C=C_{\infty}$

The subscript $w$ refer to the condition at the cylinder surface. $A, B$ and $n$ are constants.

\subsection{Dimensionless Variables}

To obtain a system of non-similar boundary layer equations applicable to the natural, mixed and forced convection regions, the following dimensionless variables are introduced

$$
\begin{array}{ll}
\eta=\frac{1}{x} P e_{x}^{1 / 2} \zeta^{-1}\left(\frac{r^{2}}{2 r_{o}}-\frac{r_{o}}{2}\right) & \zeta=\left[1+\left(\frac{R a_{x}}{P e_{x}}\right)^{1 / 2}\right]^{-1} \\
f(\zeta, \eta)=\frac{\psi(\zeta, \eta)}{\alpha r_{o} P e_{x}^{1 / 2} \zeta^{-1}} & \theta(\zeta, \eta)=\frac{T-T_{\infty}}{T_{w}-T_{\infty}}
\end{array}
$$

$$
\begin{aligned}
& \theta_{e}=\frac{T_{e}-T_{\infty}}{T_{w}-T_{\infty}}=\frac{-1}{R\left(T_{w}-T_{\infty}\right)} \quad \theta-\theta_{e}=\frac{T-T_{e}}{T_{w}-T_{\infty}} \\
& \Phi(\zeta, \eta)=\frac{C-C_{\infty}}{C_{w}-C_{\infty}}
\end{aligned}
$$

Where $\eta$ is the pseudosimilarity variable. $\zeta$ is the non-similar mixed convection parameter such that $\zeta=1$ for pure forced convection and $\zeta=$ 0 for pure natural convection. $f$ is the dimensionless stream function. $\theta$ is the dimensionless temperature. $\Phi$ is the dimensionless concentration. $\psi$ is the stream function. $\psi$ is defined by $u=(1 / r)(\partial \psi / \partial r)$ and $v=$ $-(1 / r)(\partial \psi / \partial x)$. Therefore, the continuity equation is satisfied automatically. $\theta_{e}$ is a parameter defines the variable viscosity effect. It is negative for liquids and positive for gases. If $\theta_{e}$ is large, then the effect of variable viscosity can be neglected. $R a_{x}=\left[g \beta_{T} K x\left(T_{w}-T_{\infty}\right) / \alpha v\right]$ is the local Rayleigh number. $P e_{x}=U_{\infty} x / \alpha$ is the local Peclet number. By substituting Eqs. (11) and (12) into equations of momentum, energy, concentration and boundary conditions the following non-similar dimensionless equations can be obtained:

\subsection{Dimensionless Momentum Equation}

$$
\begin{aligned}
& {\left[\theta_{e}\left(\theta-\theta_{e}\right)-2 \Gamma\left(\theta-\theta_{e}\right)^{2} f^{\prime}-M\left(\theta-\theta_{e}\right)^{2}\right] f^{\prime \prime}-\theta_{e} \theta^{\prime} f^{\prime}} \\
& -(1-\zeta)^{2}\left(\theta-\theta_{e}\right)^{2}\left[-\theta^{\prime}-N \Phi^{\prime}\right]=0
\end{aligned}
$$

where

$\Gamma=\frac{c \sqrt{K}\left(P e_{x}^{1 / 2}+R a_{x}^{1 / 2}\right)^{2} \alpha}{v x}$ is the inertia effect parameter.

$N=\frac{\beta_{C} B x^{n}}{\beta_{T} A x^{n}}$ is the Buoyancy ratio.

$M=\frac{\sigma \beta_{o}^{2} K}{\mu \phi}$ is the square of the Hartmann number.

\subsection{Dimensionless Energy Equation}

$$
\begin{aligned}
& -(2 \eta \lambda+1) \theta^{\prime \prime}+\left[-\frac{1}{2}(1+n(1-\zeta)) f-2 \lambda\right] \theta^{\prime}+n f^{\prime} \theta= \\
& -\frac{n}{2} \zeta(1-\zeta)\left(\frac{\partial f}{\partial \zeta} \theta^{\prime}-\frac{\partial \theta}{\partial \zeta} f^{\prime}\right)
\end{aligned}
$$

where

$\lambda=\frac{x}{r_{o}} \frac{1}{\left(P e_{x}^{1 / 2}+R a_{x}^{1 / 2}\right)}$ is the curvature parameter. The limit of $\lambda=0$ corresponds to vertical flat plate.

\subsection{Dimensionless Concentration Equation}

$-\frac{1}{L e}(2 \eta \lambda+1) \Phi^{\prime \prime}+\left[-\frac{1}{2}(1+n(1-\zeta)) f-\frac{2 \lambda}{L e}\right] \Phi^{\prime}+n f^{\prime} \Phi=$
$-\frac{n}{2} \zeta(1-\zeta)\left(\frac{\partial f}{\partial \zeta} \Phi^{\prime}-\frac{\partial \Phi}{\partial \zeta} f^{\prime}\right)$

where

$L e=\frac{\alpha}{D}$ is the Lewis number. 
If Eq. (13) is divided by $\theta_{e}\left(\theta-\theta_{e}\right)$ and rearranged, it can be written as follows:

$$
\begin{aligned}
& f^{\prime \prime}-2 \Gamma\left(\frac{\theta}{\theta_{e}}-1\right) f^{\prime} f^{\prime \prime}-M\left(\frac{\theta}{\theta_{e}}-1\right) f^{\prime \prime}-\frac{\frac{1}{\theta_{e}}}{\left(\frac{\theta}{\theta_{e}}-1\right)} \theta^{\prime} f^{\prime} \\
& -(1-\zeta)^{2}\left(\frac{\theta}{\theta_{e}}-1\right)\left(-\theta^{\prime}-N \Phi^{\prime}\right)=0
\end{aligned}
$$

as stated previously when $\theta_{e}$ has large value, then the effect of variable viscosity can be neglected. Therefore, if $\theta_{e} \rightarrow \infty$ and if the curvature parameter $\lambda=0$, then Eq. (16) along with Eq. (14) and (15) reduced to the equations of vertical plate with no variable viscosity effect.

\subsection{Dimensionless Boundary Conditions}

$$
\begin{array}{lll}
f(\zeta, 0)=0 & \theta(\zeta, 0)=1 & \Phi(\zeta, 0)=1 \\
f^{\prime}(\zeta, \infty)=\zeta^{2} & \theta(\zeta, \infty)=0 & \Phi(\zeta, \infty)=0
\end{array}
$$

Velocity components $u$ and $v$ along with the local Nusselt number $N u_{x}$ and local Sherwood number $S h_{x}$, in terms of dimensionless variables have the formulas:

$$
u=\frac{U_{\infty}}{\zeta^{2}} f^{\prime}
$$

$v=-\frac{r_{o}}{r} \frac{\alpha}{x} P e_{x}^{1 / 2} \frac{1}{\zeta}\left[\frac{1}{2}(1+n(1-\zeta)) f-\frac{1}{2}(1-n(1-\zeta)) \eta f^{\prime}-\right.$

$\left.\frac{1}{2} n \zeta(1-\zeta) \frac{\partial f}{\partial \zeta}\right]$

$\frac{N u_{x}}{P e_{x}^{1 / 2} \zeta^{-1}}=-\theta^{\prime}(\zeta, 0)$

$\frac{S h_{x}}{P e_{x}^{1 / 2} \zeta^{-1}}=-\Phi^{\prime}(\zeta, 0)$

Finally, the presence of $\partial / \partial \zeta$ in the above equations makes them non-similar. Furthermore, the primes denote partial differentiation with respect to $\eta$.

\section{METHOD OF SOLUTION}

In Eq. (18) $\eta \rightarrow \infty$ is replaced by a sufficiently large value (i.e. $\eta=$ $\left.\eta_{\max }\right)$ where Eq. (18) for velocity is satisfied. The domain of interest $(\zeta, \eta)$ is divided into equal spaced mesh in the $\zeta$ direction where $\Delta \zeta=$ 0.1 and another equal spaced mesh in the $\eta$ direction where $\Delta \eta=0.02$. The whole partial derivatives are approximated by the central difference formula. When $\zeta=0$ and $\zeta=1$ the governing equations are converted to similar form. The equations are non-linear, therefore, two iteration loops based on the method of successive substitutions are considered. The value of $\zeta$ is fixed in each inner iteration loop. Then on the $\eta$ domain each of Eqs. (13) to (15) is solved as a linear second order boundaryvalue problem of ordinary differential equation. The inner iteration is continued till the solution is converges. The value of $\zeta$ is advanced from 0.1 to 0.9 (mixed convection region) in the outer iteration loop. The derivatives with respect to $\zeta$ are updated after every outer iteration loop. For more details see Mohammad (2015).

\section{RESULTS AND DISCUSSION}

In order to validate the numerical results to be presented in the later sections, a comparison with previously published work on special case of the problem are conducted. The comparison of local Nusselt number for natural convection for $\lambda$ values ranged from 0.125 to 5 are shown in
Table 1. Zero curvature is related to a vertical flat plate case. The comparison show a good agreement with the previously published work.

To conserve space the effects of the parameters under consideration and mixed convection parameter $\zeta$ on the velocity, temperature and concentration profiles will be described only. It was noticed that the increase in the value of the power law index $n$ causes a decrease in the fluid velocity and the temperature and concentration gradients increases (i.e. decrease in the temperature and concentration boundary layer thicknesses). This reflect on the increasing in the convective heat and mass transfer rates as illustrated in Figure 2 and Figure 3 respectively. At a given value of $n$, as the value of the non-similarity parameter increases from 0 to 1 , the local Nusselt and Sherwood numbers decreases until it reaches a minimum value at certain $\zeta$ value and then increases. The reason of that is the definition of the local Nusselt and Sherwood numbers and does not mean that their values for combined convection is less than for natural and forced convection.

Table 1. Comparison of local Nusselt number for natural convection for a vertical cylinder. (Darcy's law, constant viscosity, $n=N=M=\Gamma=0$, $L e=1)$

\begin{tabular}{|c|c|c|c|}
\hline$\lambda$ & $\begin{array}{c}\text { Present } \\
\text { work }\end{array}$ & $\begin{array}{c}\text { Chen \& } \\
\text { Horng, (1999) }\end{array}$ & $\begin{array}{c}\text { Kumari } \text { et al., } \\
\text { (1985) see Chen } \\
\text { \& Horng, (1999) }\end{array}$ \\
\hline 0.125 & 0.4929 & 0.4942 & 0.4977 \\
\hline 0.25 & 0.5455 & 0.544 & 0.5472 \\
\hline 0.375 & 0.5995 & 0.5939 & 0.5971 \\
\hline 0.5 & 0.6537 & 0.6439 & 0.6479 \\
\hline 0.75 & 0.761 & 0.7612 & 0.7509 \\
\hline 1 & 0.8663 & 0.8669 & 0.8538 \\
\hline 1.25 & 0.9693 & 0.9705 & 0.9562 \\
\hline 1.5 & 1.07 & 1.0721 & 1.0576 \\
\hline 2 & 1.2657 & 1.2703 & 1.2571 \\
\hline 2.5 & 1.4542 & 1.4625 & 1.4519 \\
\hline 3 & 1.6367 & 1.6498 & 1.6424 \\
\hline 3.5 & 1.8373 & 1.833 & 1.829 \\
\hline 4 & 2.016 & 2.0126 & 2.012 \\
\hline 4.5 & 2.181 & 2.1891 & 2.1918 \\
\hline 5 & 2.346 & 2.3673 & 2.3688 \\
\hline
\end{tabular}

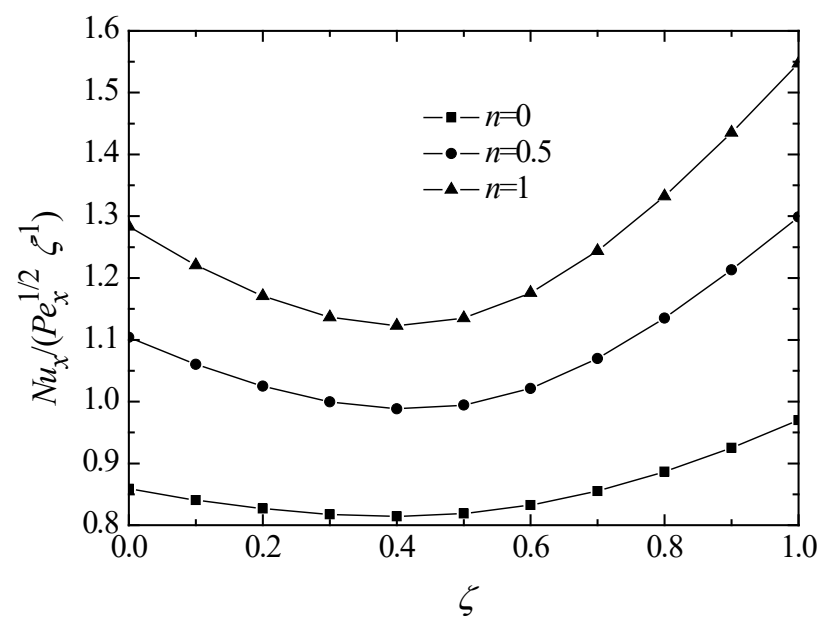

Fig. 2 Effects of power law index on the local Nusselt number for the entire regime of mixed convection. $\left(\theta_{e}=10, L e=5, N=2, \Gamma=M=\lambda=1\right)$

As the inertia effect parameter $\Gamma$ increases the velocity of the fluid decreases near the cylinder surface and increase far away for natural convection $(\zeta=0)$. However, for mixed convection $(\zeta=0.5)$ its decreases only. Furthermore, the temperature and concentration 
gradients decreases with the increasing of the value of $\Gamma$. Figure 4 and Figure 5 illustrates respectively the variation of local Nusselt and

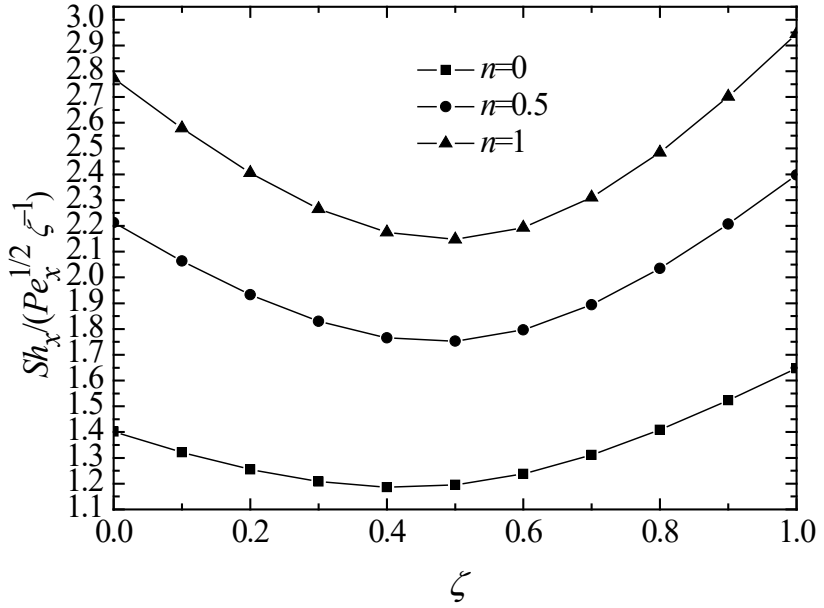

Fig. 3 Effects of power law index on the local Sherwood number for the entire regime of mixed convection. $\left(\theta_{e}=\right.$ $10, L e=5, N=2, \Gamma=M=\lambda=1$ )

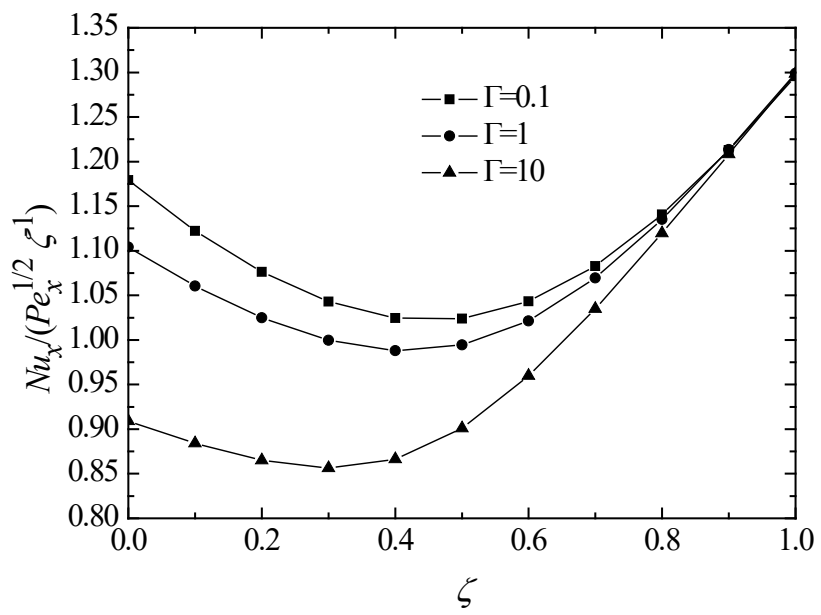

Fig. 4 Effect of inertia effect parameter on the local Nusselt Number for the entire regime of mixed convection. $\left(\theta_{e}=10, L e=5, N=2, n=0.5, M=\lambda=1\right)$

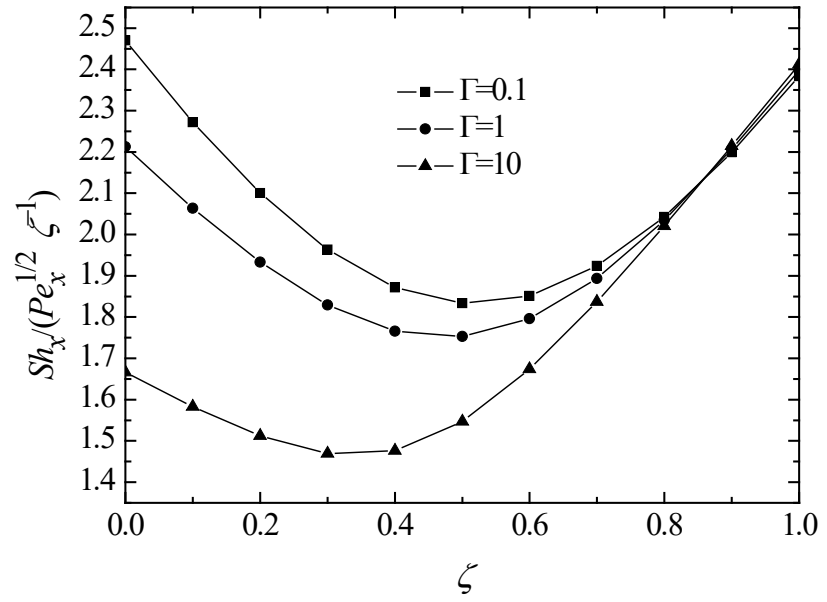

Fig. 5 Effect of inertia effect parameter on the local Sherwood Number for the entire regime of mixed convection. $\left(\theta_{e}=10, L e=5, N=2, n=0.5, M=\lambda=1\right)$

Sherwood numbers with non-similarity parameter $\zeta$ for different values of inertia effect parameter. The local Nusselt and Sherwood numbers decreases as the inertia effect parameter increases. However, this effect is small for large values of non-similarity parameter. The reason of this behavior is that, because of porous medium inertia effect additional resistance against the flow is introduced. This in turn decreasing the enthalpy of the flow streams. The model of the problem does not take into account the no-slip velocity boundary condition, therefore, the inertia of the porous medium has a little effect for the forced convection $\operatorname{limit}(\zeta=1)$

When the value of the curvature parameter $\lambda$ increases the velocity of the fluid increases and the temperature and concentration gradients decreases. Furthermore, larger values of the curvature parameter leads to higher local Nusselt and Sherwood numbers as depicted in Figure 6 and Figure 7 respectively. This denotes that a more slender cylinder, corresponding to a large value of $\left(x / r_{o}\right)$ would result in a higher heat and mass transfer rates.

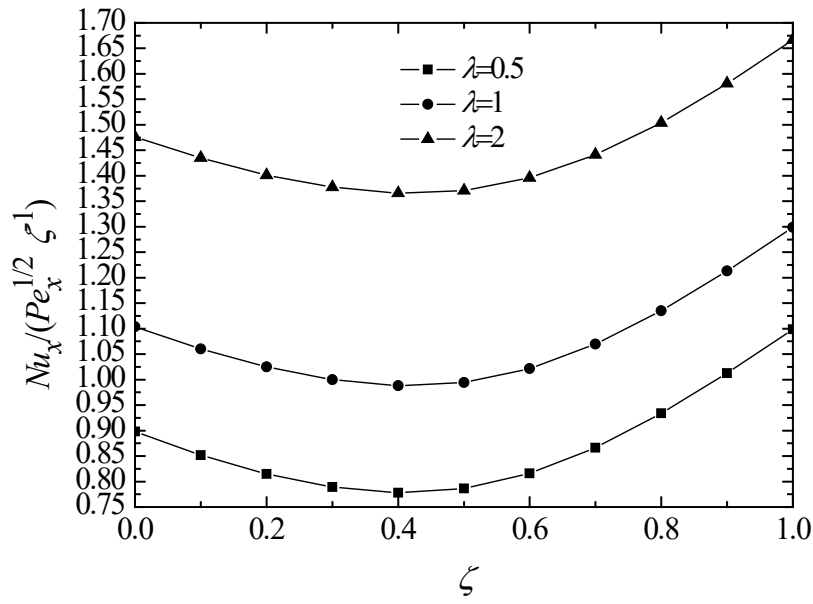

Fig. 6 Effect of curvature parameter on the local Nusselt number for the entire regime of mixed convection. $\left(\theta_{e}=\right.$ $10, L e=5, N=2, n=0.5, M=\Gamma=1$ )

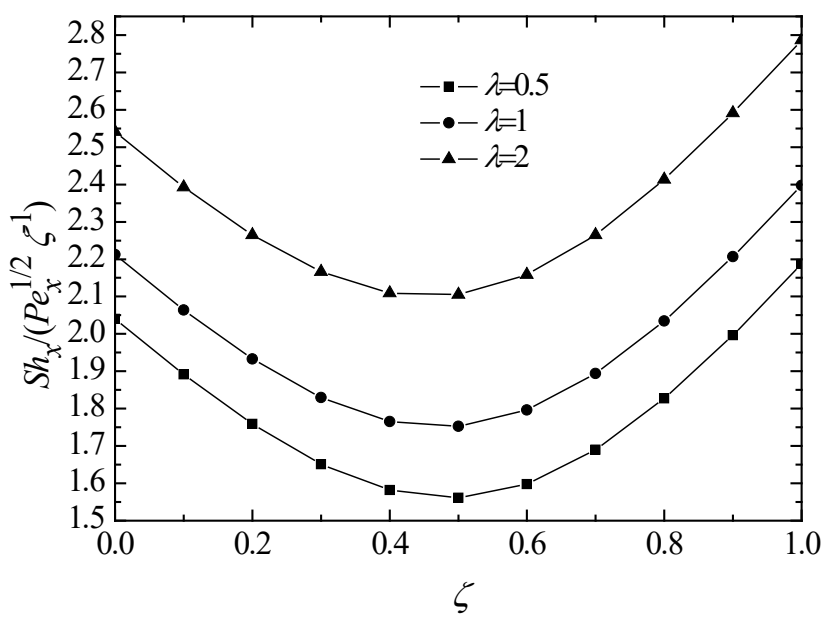

Fig. 7 Effect of curvature parameter on the local Sherwood number for the entire regime of mixed convection. $\left(\theta_{e}=\right.$ $10, L e=5, N=2, n=0.5, M=\Gamma=1$ )

Numerical results showed that increasing of the square of the Hartmann number $M$ causes reduction in the fluid velocity and temperature and concentration gradients. The resistance of the magnetic force that named as Lorentz force is the reason for the reduction in velocity of the fluid. Due to the above reasons as $M$ increases the heat and mass transfer decreases as shown in Figure 8 and Figure 9. Also, it 
is noticed from the aforementioned figures that for forced convection dominated regime, the increase in the value of $M$ do not lead to appreciable decrease in the local Nusselt and Sherwood numbers.

The effect of $N$ (the buoyancy ratio parameter) on velocity profile, temperature profile and concentration profile can be explained as follows. As $N$ increases it was noticed that the velocity increases near the cylinder surface for natural and mixed convection and decreases far away from the cylinder surface for natural convection. Also, it is observed that for natural and mixed convection the temperature and concentration decreases as $N$ increases. For forced convection the temperature and concentration are not affected by the increase in the value of $N$. Furthermore, as the mixed convection parameter increased from 0 to 1 the temperature and concentration increased and then decreased. The increase in the velocity of the fluid due to the increase in the value of buoyancy ratio parameter will cause increase in the heat and mass transfer from the surface as shown in Figure 10 and Figure 11. The behavior of the curves of local Nusselt and Sherwood numbers follow the temperature and concentration profiles.

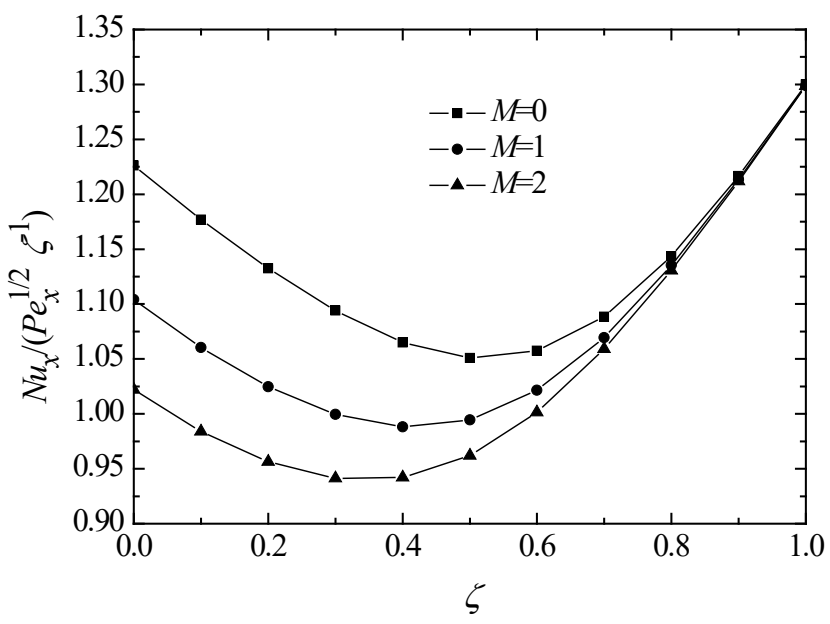

Fig. 8 Effects of the square of the Hartmann number on the Nusselt number for the entire regime of mixed convection. $\left(\theta_{e}=10, L e=5, N=2, n=0.5, \Gamma=\lambda=1\right)$

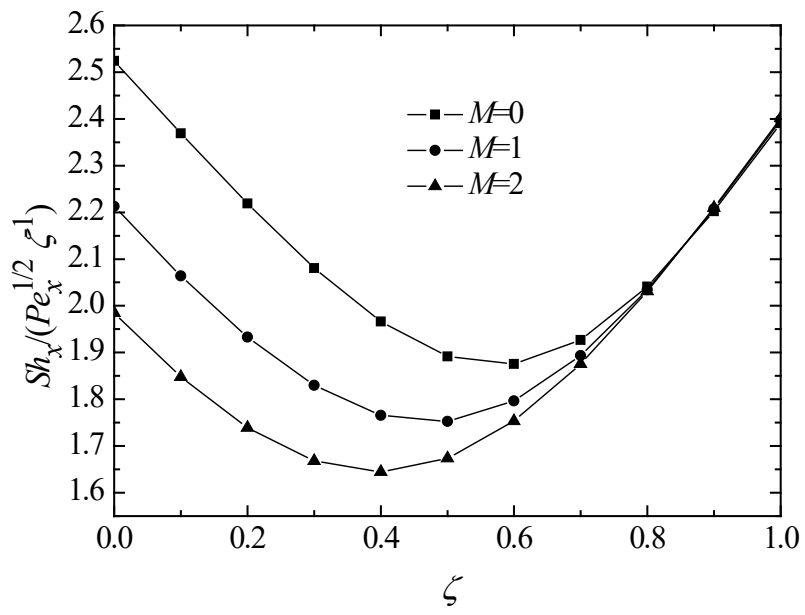

Fig. 9 Effects of the square of the Hartmann number on the Sherwood number for the entire regime of mixed convection. $\left(\theta_{e}=10, L e=5, N=2, n=0.5, \Gamma=\lambda=1\right)$

The effect of Le (Lewis number) on the velocity, temperature and concentration profiles can be summarized as follows. Increasing the value of $L e$ leads to decrease in the velocity of the fluid. Due to increase in the thermal diffusivity the thermal buoyancy forces increases and therefore the temperature of the fluid increases. On the contrary due to the decrease in the mass diffusivity, the concentration buoyancy forces decreases and thus the concentration of the fluid decreases. From Figure 12 it can be seen that the increase in $L e$ leads to decrease in the local Nusselt number. On the other hand, Figure 13 indicates that the increase in Le leads to increase in the local Sherwood number.

The effect of variable viscosity on the velocity profile, temperature profile and concentration profile are presented respectively in Figures 14, 15 and 16 . For mixed convection for gases $\left(\theta_{e}\right.$ positive) the velocity decreases as $\theta_{e}$ decreases $\left(\theta_{e} \rightarrow 0\right)$ and for liquids $\left(\theta_{e}\right.$ negative) the velocity increases as $\theta_{e}$ decreases $\left(\theta_{e} \rightarrow 0\right)$ as shown in Figure 14 Figure 15 presents that for mixed convection for gases and liquids the temperature decreases as $\theta_{e}$ decreases $\left(\theta_{e} \rightarrow 0\right)$. From Figure 16 we can see that for mixed convection for gases there are a slightly increase in the concentration due to the decrease in the $\theta_{e}$. For liquids there are a slightly decrease in the concentration due to the decrease in the $\theta_{e}$.

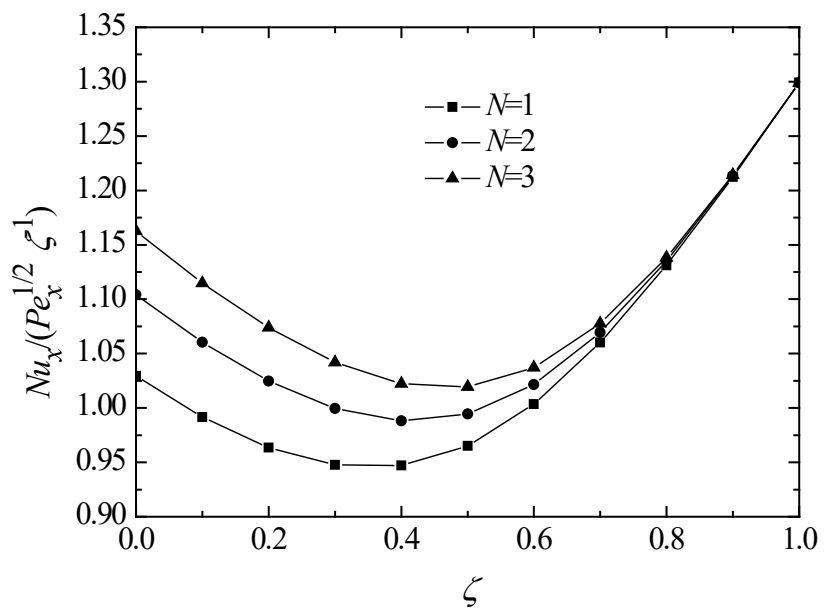

Fig. 10 Effects of buoyancy ratio on the local Nusselt number for the entire regime of mixed convection. $\left(\theta_{e}=10, L e=5\right.$, $n=0.5, \Gamma=M=\lambda=1$ ).

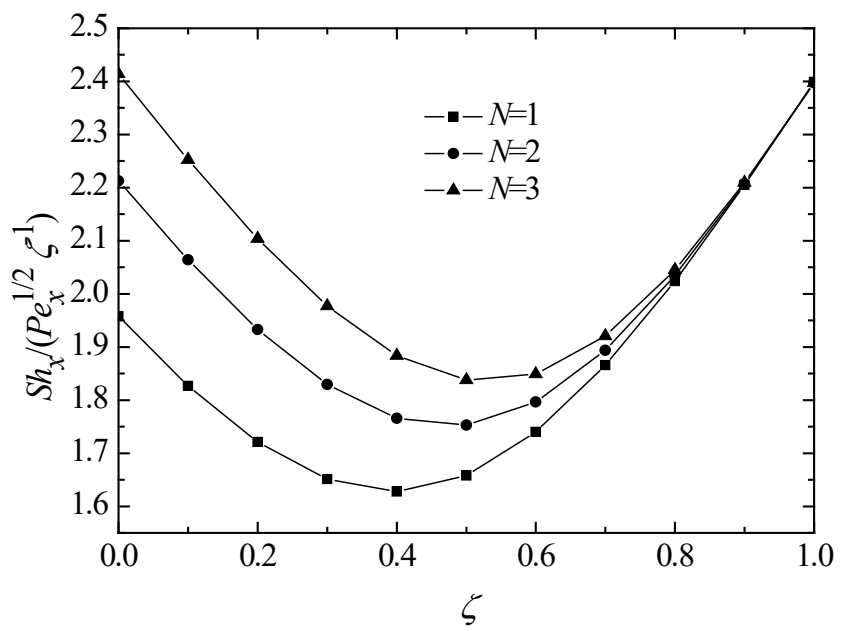

Fig. 11 Effects of buoyancy ratio on the local Sherwood number for the entire regime of mixed convection. $\left(\theta_{e}=10, L e=5, n=0.5, \Gamma=M=\lambda=1\right)$.

Figure 17 depicts the variation of local Nusselt number with the mixed convection parameter for variable viscosity. For liquids and gases the local Nusselt number increases as the value of $\theta_{e} \rightarrow 0$. Figure 18 
show that, for gases the local Sherwood number decreases as the value of $\theta_{e}$ decreases, while for liquids the value of local Sherwood number increases as the value of $\theta_{e}$ decreases.

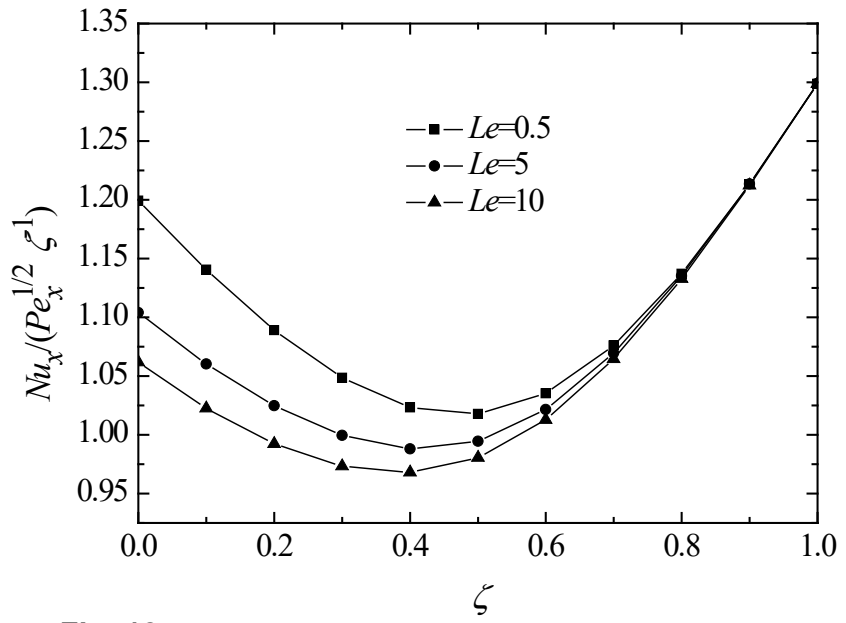

Fig. 12 Effects of Lewis number on the local Nusselt number for the entire regime of mixed convection. $\left(\theta_{e}=10, N=2\right.$, $n=0.5, \Gamma=M=\lambda=1)$.

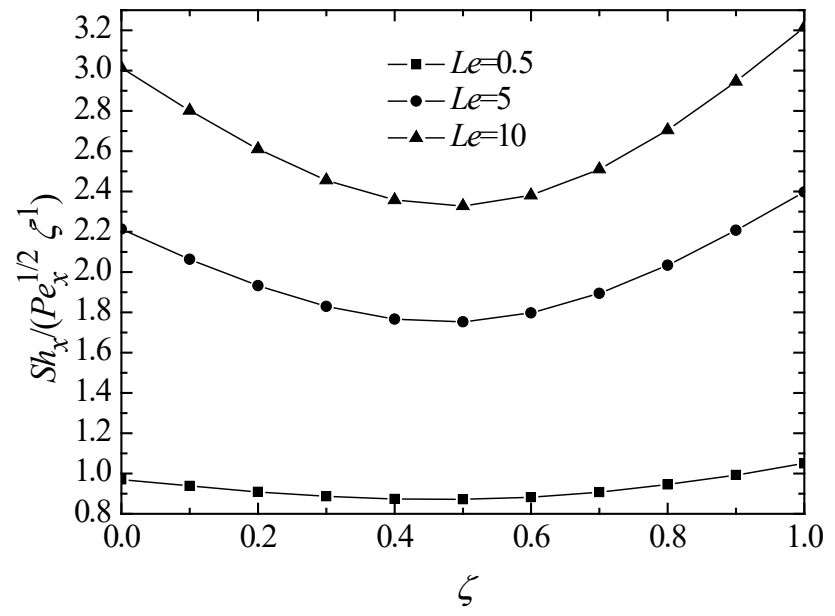

Fig. 13 Effects of Lewis number on the local Sherwood number for the entire regime of mixed convection. $\left(\theta_{e}=10\right.$, $N=2, n=0.5, \Gamma=M=\lambda=1)$.

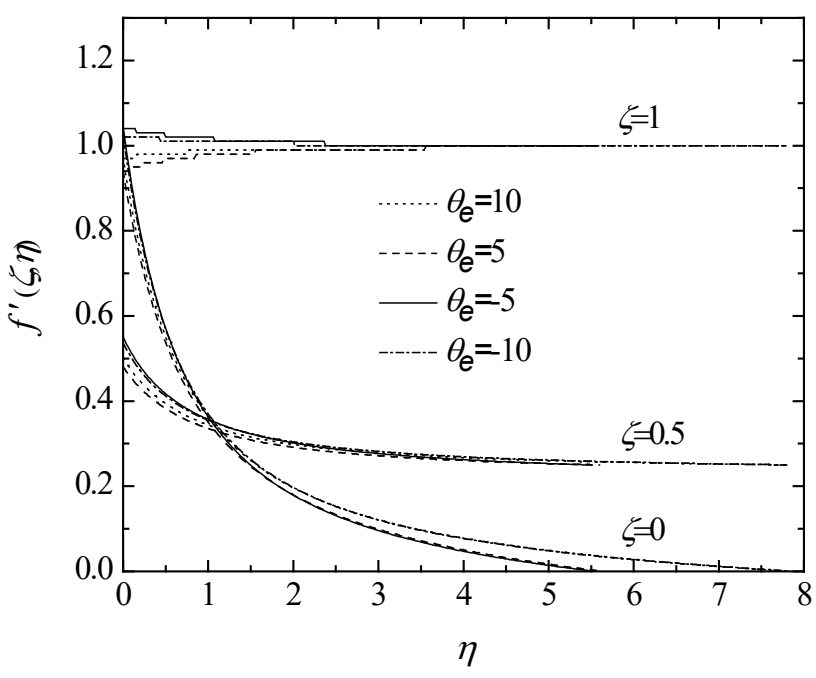

Fig. 14 Effects of variable viscosity and mixed convection parameter on the velocity profile. $(n=0.5, L e=5, N=$

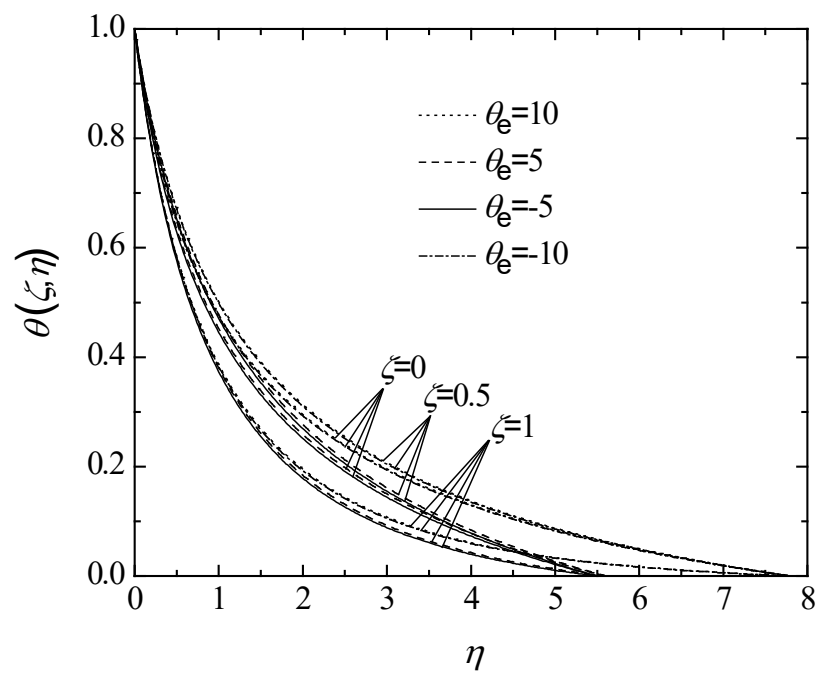

Fig. 15 Effects of variable viscosity and mixed convection parameter on the temperature profile. $(n=0.5, L e=5, N=$ $2, \Gamma=M=\lambda=1$ )

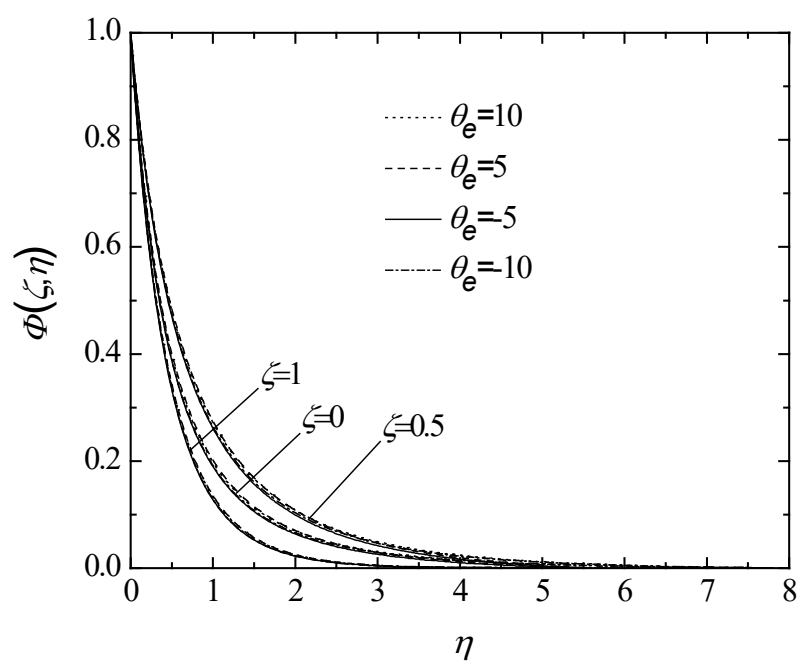

Fig. 16 Effects of variable viscosity and mixed convection parameter on the concentration profile. $(n=0.5, L e=$ $5, N=2, \Gamma=M=\lambda=1$ )

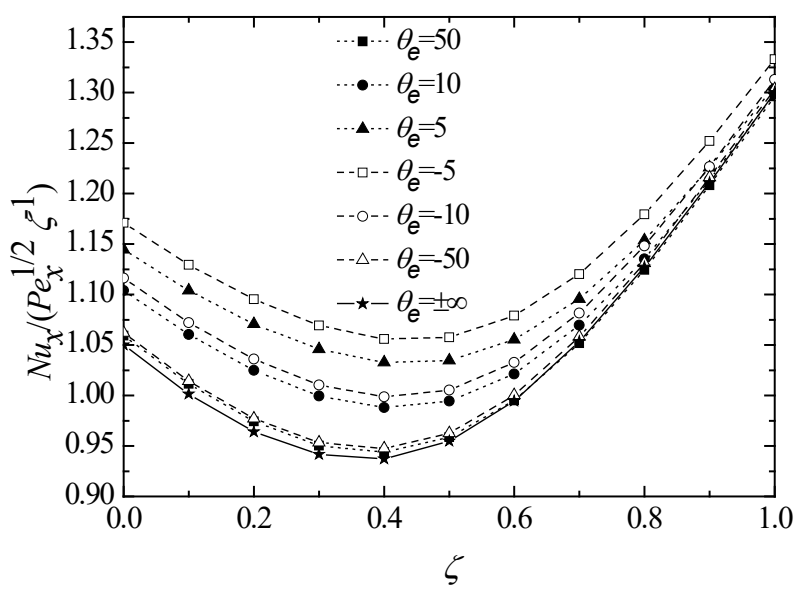

Fig. 17 Effects of variable viscosity on the local Nusselt number for the entire regime of mixed convection. $(n=$ 


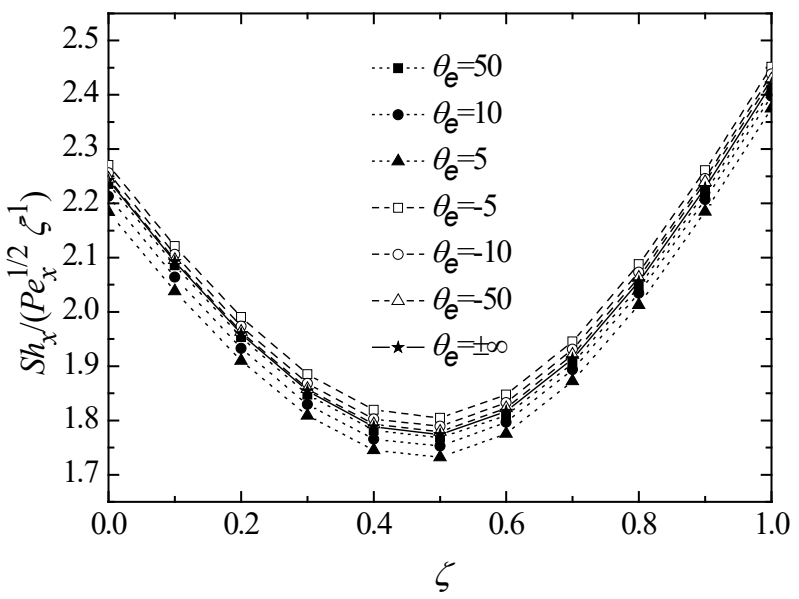

Fig. 18 Effects of variable viscosity on the local Sherwood number for the entire regime of mixed convection. $(n=0.5, L e=5, N=2, \Gamma=M=\lambda=1)$

\section{CONCLUSIONS}

This paper study the effects of variable viscosity on laminar, steady, hydromagnetic coupled heat and mass transfer by combined convection flow adjacent to a vertical cylinder embedded in a non-Darcy porous medium. The governing non-linear partial differential equations and their boundary conditions are transformed into a non-similar form by using a suitable dimensionless variables. The system of non-similar equations are solved numerically using a finite difference method. The present results of local Nusselt number are compared with previously published work on special case of the problem. The comparison is found to be in good agreement.

The study indicates that, increasing the values of power law index $n$, curvature parameter $\lambda$ and buoyancy ratio $N$ leads to increase in the rates of heat and mass transfer. The rates of heat and mass transfer decreases as the inertia effect parameter $\Gamma$ and the square of the Hartmann number $M$ increases. As the value of the Lewis number $L e$ increases the rate of heat transfer decreases while the rate of mass transfer increases. For gases and liquids the heat transfer enhanced with lower values of viscosity. The mass transfer decreases for gases and increases for liquids with lower values of viscosity.

\section{ACKNOWLEDGEMENTS}

The author would like to thank the college of engineering-university of Mosul for the support.

\section{NOMENCLATURE}

a Constant, equation 5

$A, B \quad$ Constants, equation 10

C Constant, equations 2 and 3

C Concentration $\left(\mathrm{kg} \cdot \mathrm{m}^{-3}\right)$

$d \quad$ Particle diameter $(m)$

D Mass Diffusivity $\left(\mathrm{m}^{2} \cdot \mathrm{s}^{-1}\right)$

$f \quad$ Dimensionless stream function.

$g \quad$ Gravitational acceleration $\left(m \cdot s^{-2}\right)$

$i \quad$ Index of mesh points in the $\zeta$-direction.

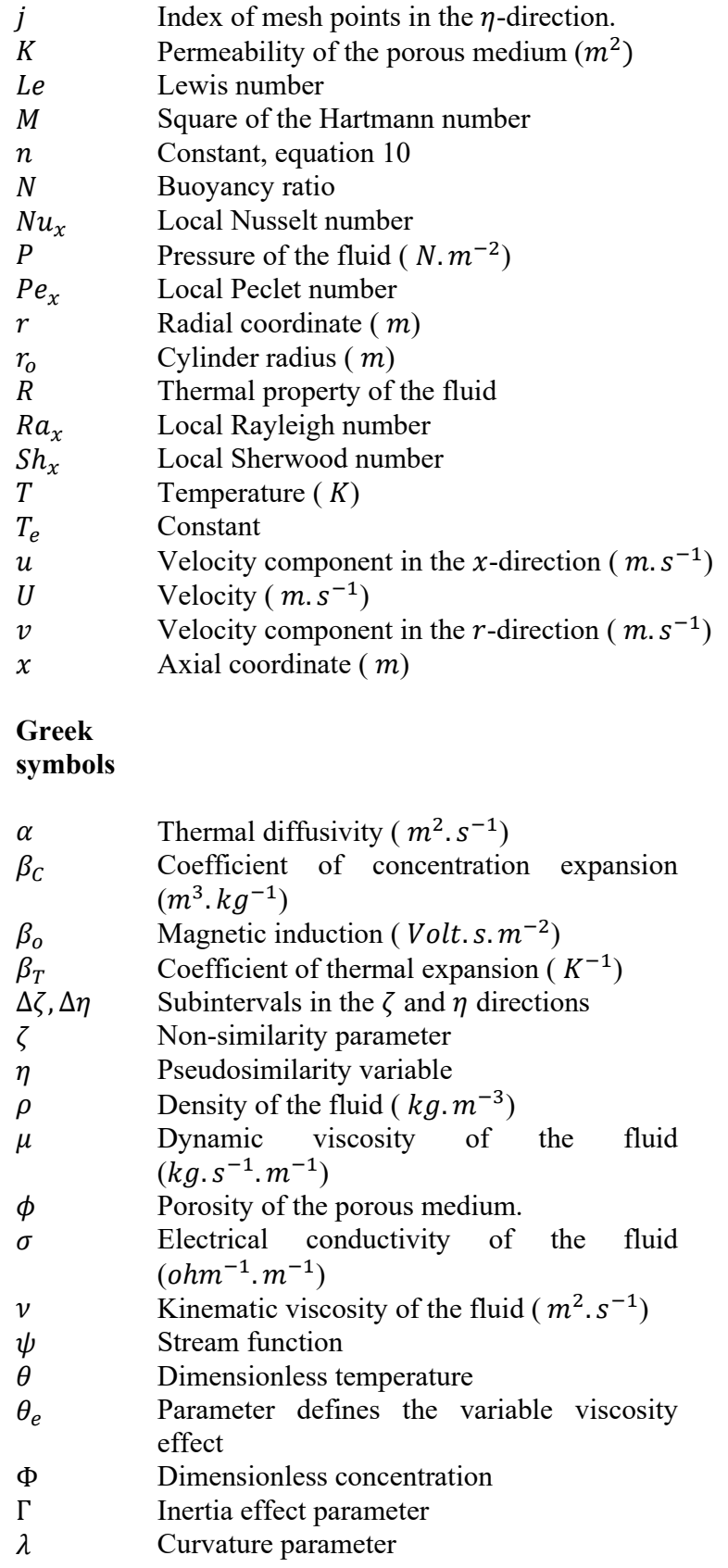

\section{Subscripts}

$\begin{array}{ll}\max & \text { Sufficiently large value } \\ w & \text { Condition at the cylinder surface } \\ \infty & \text { Free stream conditions }\end{array}$

\section{REFERENCES}

Aldoss, T. K., Jarrah, M. A., and Al-Sha'er, B. J., 1996, "Mixed Convection from a Vertical Cylinder Embedded in a Porous Medium: Non-Darcy Model," Int. J. Heat Mass Transfer, 39(6), 1141-1148. https://doi.org/10.1016/0017-9310(95)00211-1

Aldoss, T. K., 1996, "MHD Mixed Convection from a Vertical Cylinder Embedded in a Porous Medium," Int. Comm. Heat Mass Transfer, 23(4), 517-530. https://doi.org/10.1016/0735-1933(96)00036-X 
Amanulla, CH., Nagendra, N., and Suryanarayana Reddy, M., 2018, "Numerical Simulation of Slip Influence on Electric Conducting Viscoelastic Fluid Past an Isothermal Cylinder," Frontiers in Heat and Mass Transfer (FHMT), 10(10), 1-13.

http://dx.doi.org/10.5098/hmt.10.10

Bejan, A., 1995, "Convection Heat Transfer," Second Edition, John Wiley \& Sons, Inc, New York, NY, USA.

Chen, C. -H., 1998, "Mixed Convection Heat Transfer from a Horizontal Plate with Variable Surface Heat Flux in a Porous Medium," Heat and Mass Transfer, 34, 1-7. https://doi.org/10.1007/s002310050224

Chen, C. -H., and Horng, J. -H., 1999, "Natural Convection from a Vertical Cylinder in a Thermally Stratified Porous Medium," Heat and Mass Transfer, 34, 423-428. https://doi.org/10.1007/s002310050278

Chamkha, A. J., Jaradat, M., and Pop, I., 2004, "Thermophoresis Free Convection from a Vertical Cylinder Embedded in a Porous Medium," Int. J. of Applied Mechanics and Engineering, 9(3), 471-481.

Chamkha, A. J., El-Kabeir, S. M. M., and Rashad, A. M., 2011, "Heat and Mass Transfer by Non-Darcy Free Convection from a Vertical Cylinder Embedded in Porous Media with a Temperature-Dependent Viscosity," International Journal of Numerical Methods for Heat \& Fluid Flow, 21(7), 847-863. https://doi.org/10.1108/09615531111162828

El-Kabeir, S. M. M., Chamkha, A. J., and Rashad, A. M., 2014, "Effect of Thermal Radiation on Non-Darcy Natural Convection from a Vertical Cylinder Embedded in a Nanofluid Porous Media," Journal of Porous Media, 17(3), 269-278.

https://doi.org/10.1615/JPorMedia.v17.i3.70

Ferdows, M., Hamad, M. A. A., and Ali, M., 2015, "Lie Group Analysis on Brownian Motion and Thermophoresis Effect on Free Convective Boundary-Layer Flow on a Vertical Cylinder Embedded in a NanofluidSaturated Porous Medium," Hindawi Publishing Corporation, Journal of Applied Mathematics, 2015, Article ID 741352, 1-6.

http://dx.doi.org/10.1155/2015/741352

Flilihi, E., Sriti, M., and Achemlal, D., 2019, "Numerical Solution on Non-uniform Mesh of Darcy-Brinkman-Forchheimer Model for Transient Convective Heat Transfer over Flat Plate in Saturated Porous Medium," Frontiers in Heat and Mass Transfer (FHMT), 12(12), 1-10. http://dx.doi.org/10.5098/hmt.12.12

Gorla, R. S. R., and Hossain, A., 2013, "Mixed Convection Boundary Layer Flow over a Vertical Cylinder Embedded in a Porous Medium Saturated with a Nanofluid," International Journal of Numerical Methods for Heat \& Fluid Flow, 23(8), 1393-1405.

https://doi.org/10.1108/HFF-03-2012-0064

Ganapathirao, M., Chamkha, A. J., and Srinivasa Rao, N., 2019, "Effects of Non-uniform Slot Suction/Injection and Chemical Reaction on Mixed Convective MHD Flow Along a Vertical Wedge Embedded in a Porous Medium," Frontiers in Heat and Mass Transfer (FHMT), 13(15), 1-13. http://dx.doi.org/10.5098/hmt.13.15

Hooper, W. B., Chen, T. S., and Armaly, B. F., 1994, "Mixed Convection Along an Isothermal Vertical Cylinder in Porous Media," Journal of Thermophysics and Heat Transfer, 8(1), 92-99.

https://doi.org/10.2514/3.505
Hossain, M. A., Vafai, K., and Khanafer, K. M. N., 1999, "Non-Darcy Natural Convection Heat and Mass Transfer Along a Vertical permeable Cylinder Embedded in a porous Medium," Int. J. Therm. Sci., 38, 854862.

https://doi.org/10.1016/S1290-0729(99)80040-4

Jayanthi, S., and Kumari, M., 2006, "Effect of Variable Viscosity on Non-Darcy Free or Mixed Convection Flow on a Vertical Surface in a Fluid Saturated Porous Medium," Mechanics Research Communications, 33(2), 148-156. https://doi.org/10.1016/j.mechrescom.2005.09.001

Jafarian, B., Hajipour, M., and Khademi, R., 2016, "Conjugate Heat Transfer of MHD Non-Darcy Mixed Convection Flow of a Nanofluid over a Vertical Slender Hollow Cylinder Embedded in Porous Media," Trans. Phenom. Nano Micro Scales, 4(1), 1-10. https://doi.org/10.7508/tpnms.2016.01.001

Kumari, M., Nath, G., and Pop, I., 1993, "Non-Darcy Mixed Convection Flow with Thermal Dispersion on a Vertical Cylinder in a Saturated Porous Medium," Acta Mechanica, 100, 69-77.

https://doi.org/10.1007/BF01176862

Kumari, M., Bercea, C., and Pop, I., 2007, "Effect of Non-uniform Suction or Injection on Mixed Convection Flow over a Vertical Cylinder Embedded in a Porous Medium," Malaysian Journal of Mathematical Sciences, 1(2), 193-204.

Merkin, J. H., and Pop, I., 1987, "Mixed Convection Boundary-Layer on a Vertical Cylinder Embedded in a Saturated Porous Medium," Acta Mechanica, 66, 251-262.

https://doi.org/10.1007/BF01184297

Mohammad, S. A., 2015, "Influence of Radiation on Non-Darcy Mixed Convection Along a Non-isothermal Vertical Plate in a Porous Medium with Thermal Dispersion and Viscous Dissipation," Al-Rafidain Engineering, 23, 12-28.

https://doi.org/10.33899/rengj.2015.101032

Mohammad, S. A., 2019, "Heat and Mass Transfer by Hydromagntic Mixed Convection Flow Along a Vertical Cylinder Embedded in a NonDarcy Porous Medium with Heat Source," International Journal of Mechanical and Production Engineering Research and Development (IJMPERD), 9(5), 171-182.

Paper Id.: IJMPERDOCT201916

Richardson, L., and Straughan, B., 1993, "Convection with Temperature Dependent Viscosity in a Porous Medium: Nonlinear Stability and the Brinkman Effect," Atti della Accademia Nazionale dei Lincei. Classe di Scienze Fisiche, Matematica e Naturali. Rendiconti Lincei. Matematica e Applicazioni, serie 9, 4(3), 223-230.

http://eudml.org/doc/244260

Rani, H. P., and Kim, C. N., 2008, “Transient Free Convection Flow over an Isothermal Vertical Cylinder with Temperature Dependent Viscosity," Korean J. Chem.Eng., 25(1), 34-40.

https://doi.org/10.1007/s11814-008-0006-5

Rohni, A. M., Ahmad, S., Merkin, J. H., and Pop, I., 2013, "Mixed Convection Boundary-Layer Flow Along a Vertical Cylinder Embedded in a Porous Medium Filled by a Nanofluid," Transp Porous Med, 96, 237-253.

https://doi.org/10.1007/s11242-012-0085-y

Rashad, A. M., Abbasbandy, S., and Chamkha, A. J., 2014, "Non-Darcy Natural Convection from a Vertical Cylinder Embedded in a Thermally 
Stratified and Nanofluid-Saturated Porous Media," Transactions of the ASME, Journal of Heat Transfer, 136(2), (022503-1)-(022503-9).

https://doi.org/10.1115/1.4025559

Reddy, M. G., 2014, "Radiation Effects on MHD Natural Convection Flow Along a Vertical Cylinder Embedded in a Porous Medium with Variable Surface Temperature and Concentration," Frontiers in Heat and Mass Transfer (FHMT), 5(4), 1-9.

http://dx.doi.org/10.5098/hmt.5.4

Shakeri, E., Nazari, M., and Kayhani, M. H., 2012, "Free Convection Heat Transfer over a Vertical Cylinder in a Saturated Porous Medium Using a Local Thermal Non-equilibrium Model," Transp. Porous Med., 93, 453-460. https://doi.org/10.1007/s11242-012-9962-7
Shu, J. -J., Wang, Q. -W., and Pop, I., 2017, "Dual Solutions for Opposing Mixed Convection in Porous Media," Journal of Heat Transfer-Transactions of the ASME, 139(10), 102-105. https://doi.org/10.1115/1.4036727

Takhar, H. S., Chamkha, A. J., and Nath, G., 2002, "Natural Convection on a Vertical Cylinder Embedded in a Thermally Stratified High-Porosity Medium," International Journal of Thermal Sciences, 41, 83-93. https://doi.org/10.1016/S1290-0729(01)01306-0

Vasu, B., Gorla, R. S. R., Prasad, V. R., and Beg, O. A., 2017, “Chemical Reaction on Heat and Mass Flow Past a Vertical Cylinder Embedded in Non-Darcy Porous Medium," Heat and Mass Transfer Research Journal, 1(1), 1-24.

http://www.cansrg.com/journals/hmtrj/2017/Vasu-et-al/index.html 